\title{
Experimental Mathematics
}

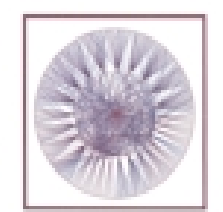

\section{Testing the Logarithmic Comparison Theorem for Free Divisors}

\section{F. J. Castro-Jiménez \& J. M. Ucha-Enríquez}

To cite this article: F. J. Castro-Jiménez \& J. M. Ucha-Enríquez (2004) Testing the Logarithmic Comparison Theorem for Free Divisors, Experimental Mathematics, 13:4, 441-449, DOI: 10.1080/10586458.2004.10504553

To link to this article: https://doi.org/10.1080/10586458.2004.10504553

Published online: 03 Apr 2012.

Submit your article to this journal $₫$

Џll Article views: 16

Q View related articles $\leftarrow$

4 Citing articles: 6 View citing articles 


\title{
Testing the Logarithmic Comparison Theorem for Free Divisors
}

\author{
F. J. Castro-Jiménez and J. M. Ucha-Enríquez
}

\section{CONTENTS}

1. Introduction

2. Spencer Divisors

3. How to Deduce that LCT Holds

4. How to Deduce that LCT Does Not Hold

5. On the Regularity of Logarithmic $\mathcal{D}$-Modules

6. Conclusions

Acknowledgments

References

2000 AMS Subject Classification: Primary 14F50;

Secondary $32 \mathrm{C} 38,32 \mathrm{C} 35,13 \mathrm{Pxx}, 68 \mathrm{~W} 30$

Keywords: de Rham cohomology, Logarithmic Comparison Theorem, free divisors, Gröbner bases

\begin{abstract}
We propose in this work a computational criterion to test if a free divisor $D \subset \mathbf{C}^{n}$ verifies the Logarithmic Comparison Theorem (LCT); that is, whether the complex of logarithmic differential forms computes the cohomology of the complement of $D$ in $\mathbf{C}^{n}$.

For Spencer free divisors $D \equiv(f=0)$, we solve a conjecture about the generators of the annihilating ideal of $1 / f$ and make a conjecture on the nature of Euler homogeneous free divisors which verify LCT. In addition, we provide examples of free divisors defined by weighted homogeneous polynomials that are not locally quasi-homogeneous.
\end{abstract}

\section{INTRODUCTION}

Let $D$ be a divisor (i.e., a hypersurface) in $X:=\mathbf{C}^{n}$. $\mathrm{K}$. Saito introduced in [Saito 80 ] the complex $\Omega^{\bullet}(\log D)$ of holomorphic differential forms with logarithmic poles along $D$. It is a subcomplex of the meromorphic de Rham complex $\Omega^{\bullet}(\star D)$ of meromorphic differential forms with poles along $D$. Let us denote by $i_{D}$ the inclusion morphism $\Omega^{\bullet}(\log D) \hookrightarrow \Omega^{\bullet}(\star D)$. Grothendieck's Comparison Theorem [Grothendieck 66] proves that the last complex calculates the cohomology of the complement of $D$ in $X$. It was proved in [Castro et al. 96] that if $D$ is a locally quasi-homogeneous free divisor then the Logarithmic Comparison Theorem (LCT) holds for $D$. We claim that LCT holds for $D$ if the morphism $i_{D}$ is a quasi-isomorphism, i.e., if $i_{D}$ induces an isomorphism on cohomology.

Let us denote by $\mathcal{O}=\mathcal{O}_{X}$ the sheaf of holomorphic functions on $X$ and take $x \in X$. Denote by $\operatorname{Der}\left(\mathcal{O}_{x}\right)$ the $\mathcal{O}_{x}$-module of $\mathbf{C}$-derivations of $\mathcal{O}_{x}$ (the elements in $\operatorname{Der}\left(\mathcal{O}_{x}\right)$ are called vector fields). This yields the sheaf $\operatorname{Der}(\mathcal{O})$ of vector fields on $X$.

Following K. Saito [Saito 80], a vector field $\delta \in$ $\operatorname{Der}\left(\mathcal{O}_{x}\right)$ is said to be logarithmic with respect to $D$ if $\delta(f)=a f$ for some $a \in \mathcal{O}_{x}$, where $f$ is a local (reduced) equation of the germ $(D, x) \subset(X, x)$. The $\mathcal{O}_{x}$-module

(C) A K Peters, Ltd. 1058-6458/20045 0.50 per page Experimental Mathematics 13:4, page 441 
of logarithmic vector fields (or logarithmic derivations) with respect to $D$ is denoted by $\operatorname{Der}(-\log D)_{x}$ and it is closed under the bracket product $[-,-]$. This yields a $\mathcal{O}$ module coherent sheaf denoted by $\operatorname{Der}(-\log D)$, which is a submodule of the sheaf of vector fields over $X$.

The divisor $D$ is said to be free at the point $x \in D$ (see [Saito 80]) if the $\mathcal{O}_{x}$-module $\operatorname{Der}(-\log D)_{x}$ is free (and, in this case, of rank $n$ ). The divisor $D$ is called free if it is free at each point $x \in D$. Saito's criterion ([Saito 80]) says that a divisor $D \equiv(f=0)$ is free at a point $x \in D$ if and only if there exists a basis $\left\{\delta_{1}, \ldots, \delta_{n}\right\}$ of $\operatorname{Der}(-\log D)_{x}$ whose determinant of coefficients with respect to the partial derivatives is equal to $u \cdot f$, for some $u \in \mathcal{O}_{x}$ such that $u(x) \neq 0$. Smooth divisors and normal crossing divisor are free. By [Saito 80], any plane curve $D \subset \mathbf{C}^{2}$ is a free divisor.

As stated in [Castro et al. 96], a divisor $D \subset \mathbf{C}^{n}$ is called locally quasi-homogeneous if for each $x \in D$ there exists a system of local coordinates $\left(z_{1}, \ldots, z_{n}\right)$ around $x$ such that the germ $(D, x)$ is defined by a weighted homogeneous polynomial $h\left(z_{1}, \ldots, z_{n}\right)$ with strictly positive weights for the variables $z_{i}$. If a plane curve $D \subset \mathbf{C}^{2}$ is defined by a weighted homogeneous polynomial, then $D$ is a locally quasi-homogeneous free divisor-so LCT holds for such a plane curve. In [Calderón et al. 02] a converse of this last result is proved: if $D$ is a plane curve and LCT holds for $D$, then $D$ is locally quasi-homogeneous. It was also shown in [Calderón et al. 02] that, in dimension 3 , there are free divisors that verify LCT and are not locally quasi-homogeneous.

A divisor $D \subset X$ is said to be Euler homogeneous if for each $x \in D$ there exist a local equation $f$ of the germ $(D, x)$ and a vector field $\delta \in \operatorname{Der}\left(\mathcal{O}_{x}\right)$ vanishing at $x$ such that $\delta(f)=f$. In [Calderón et al. 02], it is proved that LCT implies Euler homogeneity for place curves.

It is an open problem to describe which free divisors verify LCT in dimension greater than 2, and there is an open conjecture related to this problem:

Conjecture 1.1. ([Torrelli 04]) LCT holds for the germ $(D, x)$ if and only if the annihilator ideal $A n n_{\mathcal{D}_{x}}(1 / f)$ is generated by elements of order 1, where $f$ is a local equation of the germ $(D, x)$.

Here $\mathcal{D}_{x}$ stands for the ring of germs of linear differential operators with coefficients in the ring $\mathcal{O}_{x}$ and $A n n_{\mathcal{D}_{x}}(1 / f)$ for the ideal of linear differential operators $P$ in $\mathcal{D}_{x}$ such that $P(1 / f)=0$. The order of a nonzero element $P$ of $\mathcal{D}_{x}$,

$$
P=\sum_{\alpha=\left(\alpha_{1}, \ldots, \alpha_{n}\right) \in \mathbf{N}^{n}} a_{\alpha} \partial^{\alpha},
$$

is the integer $\operatorname{ord}(P)=\max \left\{|\alpha|=\alpha_{1}+\cdots+\alpha_{n} \mid a_{\alpha} \neq 0\right\}$ (here $a_{\alpha} \in \mathcal{O}_{x}$ and $\partial_{i}$ stands for the partial derivative $\left.\frac{\partial}{\partial x_{i}}\right)$. The principal symbol of $P$ is by definition the expression

$$
\sigma(P)=\sum_{\alpha,|\alpha|=\operatorname{ord}(P)} a_{\alpha} \xi^{\alpha},
$$

viewed as an element of the ring of polynomials $\mathcal{O}_{x}[\xi]$ in $n$ variables, $\xi=\left(\xi_{1}, \ldots, \xi_{n}\right)$, with coefficients in $\mathcal{O}_{x}$. If $\mathcal{D}=$ $\mathcal{D}_{X}$ is the sheaf of rings of linear differential operators with holomorphic coefficients, the stalk of $\mathcal{D}$ at $x$ is $\mathcal{D}_{x}$. The ring $\mathcal{D}_{x}$ is filtered by the order of its elements. The associated graded ring is denoted by $\operatorname{gr}\left(\mathcal{D}_{x}\right)$. It is easy to prove that $\operatorname{gr}\left(\mathcal{D}_{x}\right)$ is isomorphic to $\mathcal{O}_{x}[\xi]$.

One of the difficulties in the study of which divisors $D$ verify LCT is addressed by the examples. Given a divisor $D$ it is hard to prove whether the inclusion morphism $i_{D}$ is a quasi-isomorphism ${ }^{1}$. We propose here a computational tool to test LCT in the free case.

An essential ingredient for our method to cover the case of free divisors is a recent result of [Calderón and Narváez 04]: if $D$ is a free divisor that verifies LCT then $D$ is a Spencer divisor. Roughly speaking, $D$ is a Spencer free divisor (see below) if it is free and admits a special free resolution for the $\mathcal{D}$-module $\mathcal{D} /\langle\operatorname{Der}(-\log D)\rangle$ where $\langle\operatorname{Der}(-\log D)\rangle$ is the left ideal of $\mathcal{D}$ generated by $\operatorname{Der}(-\log D)$. With the help of this result, it is enough to provide a criterion to test LCT for Spencer-free divisors; the non-Spencer ones do not verify LCT.

\section{SPENCER DIVISORS}

In order to allow an easier reading of this article we review here the results of [Castro and Ucha 02, Sections 3 and 4].

In [Calderón 99] the author associates with a free divisor $D \subset \mathbf{C}^{n}$ the so-called augmented Spencer logarithmic complex

$$
\mathcal{D} \otimes_{\mathcal{O}} \wedge^{\bullet} \operatorname{Der}(-\log D) \rightarrow M^{\log D} \rightarrow 0 .
$$

It is a complex of $\mathcal{D}$-modules and $M^{\log D}$ stands for the quotient $\frac{\mathcal{D}}{\langle\operatorname{Der}(-\log D)\rangle}$.

Definition 2.1. ([Castro and Ucha 02, Definition 3.3]) We say that a free divisor $D$ is a Spencer divisor if

\footnotetext{
${ }^{1}$ The reader can consider, for example, the ad hoc explicit proof in [Calderón et al. 02] to show that the divisor $(x y(x+y)(x z+y)=$ 0) $\subset \mathbf{C}^{3}$ verifies LCT.
} 
the $\mathcal{D}$-module $M^{\log D}$ is holonomic and the augmented Spencer logarithmic complex is a (locally) free resolution of $M^{\log D}$.

A coherent $\mathcal{D}$-module $M$ on $X$ is said to be holonomic if its characteristic variety (see [Mebkhout 89 , Chapter I, (2.2)]) has dimension $n$.

Definition 2.2. ([Calderón 99, Definition 4.1.1]) The divisor $D$ is said to be Koszul free at the point $x \in D$ if it is free at $x$ and there exists a basis $\left\{\delta_{1}, \ldots, \delta_{n}\right\}$ of $\operatorname{Der}(-\log D)_{x}$ such that the sequence $\left\{\sigma\left(\delta_{1}\right), \ldots, \sigma\left(\delta_{n}\right)\right\}$ of principal symbols is a regular sequence in the ring $\operatorname{gr}\left(\mathcal{D}_{x}\right)$. The divisor $D$ is Koszul free if it is Koszul free at any point of $D$.

By [Saito 80] and [Calderón 99, 4.2.2.] any plane curve $D \subset \mathbf{C}^{2}$ is a Koszul free divisor. By [Calderón 99, Propositions 4.1.2 and 4.1.3] if $D$ is Koszul free (in particular if $D$ is a plane curve) then it is a Spencer divisor, but the converse is not true, see [Calderón 99, Remark 4.2.4] and [Castro and Ucha 02, Section 5.3].

Given a coherent $\mathcal{D}$-module $M$, the solution complex of $M$ is by definition the complex $\mathbf{R} \mathcal{H o m}_{\mathcal{D}}(M, \mathcal{O}$ ) (see, for example,. [Mebkhout 89, Chapter I, (2.6)]). This complex will be simply denoted by $\operatorname{Sol}(M)$.

The following proposition is a consequence of [Calderón 99, Theorem 4.2.1].

Proposition 2.3. If $D$ is a Spencer divisor then there exists a natural quasi-isomorphism from $\mathrm{Sol}\left(M^{\log }\right)$ to $\Omega^{\bullet}(\log D)$.

For each $x \in X$, we can consider the ideal $A n n_{\mathcal{D}_{x}}^{(1)}(1 / f)$ (here, $f$ is a local equation of the germ $(D, x)$ ) generated by the differential operators $P \in \mathcal{D}_{x}$ such that $P(1 / f)=0$ and the order of $P$ is equal to 1 . In fact, such an operator $P$ must have the form $\delta+a$ where $\delta$ is a logarithmic derivation in $\operatorname{Der}(-\log D)_{x}$ and $\delta(f)=a f$ with $a \in \mathcal{O}_{x}$.

Let us denote by $\widetilde{M}^{\log D}$ the quotient $\mathcal{D}$-module with stalks

$$
\left(\widetilde{M}^{\log D}\right)_{x}:=\frac{\mathcal{D}_{x}}{A n n_{\mathcal{D}_{x}}^{(1)}(1 / f)} .
$$

The module $\widetilde{M}^{\log D}$ admits in the Spencer case a free resolution completely analogous to the one of $M^{\log D}$ :

$$
\mathcal{D} \otimes_{\mathcal{O}} \wedge^{\bullet} \widetilde{\operatorname{Der}}(-\log D) \rightarrow \widetilde{M}^{\log D} \rightarrow 0,
$$

where $\widetilde{\operatorname{Der}}(-\log D)$ denotes the free $\mathcal{O}$-module of differential operators that can be written locally as $\delta+a$ such that $\delta \in \operatorname{Der}(-\log D)$ and $\delta(f)=a f$ for some holomorphic germ $a$.

The following theorem is proved in [Castro and Ucha 02, Theorem 4.3]:

Theorem 2.4. For each Spencer divisor $D \subset X$ we a have an isomorphism $\left(M^{\log D}\right)^{*} \simeq \widetilde{M}^{\log D}$.

In this theorem $(-)^{*}$ means duality in the sense of $\mathcal{D}$ module theory (see, for example, [Mebkhout 89, Chapter I, (4.1) ]).

These last two results allow us to use $\mathcal{D}$-module theory in connection with the logarithmic comparison theorem as we will show in the following sections. The following question is open:

Problem 2.5. Identify which free divisors are Spencer divisors.

An example of a free divisor that is not Spencer is given in [Calderón and Narváez 04].

Remark 2.6. For a given divisor $D \equiv(f=0)$ with $f \in R=\mathbf{C}\left[x_{1}, \ldots, x_{n}\right]$ the modules $M^{\log D}$ and $\widetilde{M}^{\log D}$ can be obtained by means of computations of syzygies of polynomials using Gröbner bases: if

$$
\left(a_{1} \partial_{1}+\cdots+a_{n} \partial_{n}\right)(f)=m f
$$

for some $m \in R$, then

$$
a_{1} \frac{\partial f}{\partial x_{1}}+\cdots+a_{n} \frac{\partial f}{\partial x_{n}}-m f=0 .
$$

Since the inclusion of the ring of differential operators with coefficients in $R$ - the Weyl algebra-in $\mathcal{D}_{x}$ is flat, these computations in $R$ cover the analytical setting.

\section{HOW TO DEDUCE THAT LCT HOLDS}

Let $D \subset \mathbf{C}^{n}$ be a free divisor. We state the first criterion to prove that LCT holds. In the proof of its correctness, we use some conditions that are sufficient for the complexes $\Omega^{\bullet}(\log D)$ and $\Omega^{\bullet}(\star D)$ to be quasi-isomorphic ${ }^{2}$. For each germ $(D, x) \subset\left(\mathbf{C}^{n}, x\right)$ defined by a holomorphic function $f$, we denote by $b_{f}$ the $b$-function associated with $f$ (see [Bernstein 72]). Let us denote by $\phi_{D}: \widetilde{M}^{\log D} \rightarrow \mathcal{O}(\star D)$ the natural morphism defined by $\phi_{D}(\bar{P})=P\left(\frac{1}{f}\right)$. For any coherent $\mathcal{D}$-module $M$ the

\footnotetext{
${ }^{2}$ The symbol $\simeq$ between complexes stands naturally for quasiisomorphisms from now on.
} 
de Rham complex $D R(M)$ associated with $M$ is by definition the complex of sheaves of $\mathbf{C}$-vector spaces

$$
0 \rightarrow M \stackrel{\nabla}{\rightarrow} M \otimes_{\mathcal{O}} \Omega^{1} \stackrel{\nabla}{\rightarrow} \ldots \stackrel{\nabla}{\rightarrow} M \otimes_{\mathcal{O}} \Omega^{n} \rightarrow 0,
$$

where $\Omega^{i}$ stands for the sheaf of holomorphic differential $i$-forms on $X$ and $\nabla$ is the exterior derivative defined by $\nabla(m \otimes \omega)=(\nabla m) \wedge \omega-(-1)^{\operatorname{deg}(w)} m \otimes d \omega$.

Criterion 3.1. If $D$ is a Spencer divisor, then

$$
A n n_{\mathcal{D}}(1 / f)=A n n_{\mathcal{D}}^{(1)}(1 / f) \Rightarrow \text { LCT holds for } D \text {. }
$$

Proof: Let us suppose that $D$ is Spencer. On the one hand, we have:

- By Proposition 2.3,

$$
\Omega^{\bullet}(\log D) \simeq \operatorname{Sol}\left(M^{\log D}\right)
$$

and (see [Mebkhout 89, page 41]) $\operatorname{Sol}\left(M^{\log D}\right) \simeq$ $D R\left(\left(M^{\log D}\right)^{*}\right)$.

- By Theorem 2.4, we deduce that

$$
D R\left(\left(M^{\log D}\right)^{*}\right) \simeq D R\left(\widetilde{M}^{\log D}\right) .
$$

On the other hand, -1 is the smallest integer root of the $b$-function $b_{f}$ (see [Torrelli 04, Proposition 1.3]), and then we have

$$
\mathcal{D} \cdot \frac{1}{f}=\mathcal{O}(\star D),
$$

where $\mathcal{O}(\star D)$ is the sheaf of meromorphic functions with poles along $D$ and $\mathcal{D} \cdot \frac{1}{f}$ is its sub- $\mathcal{D}$-module generated (locally) by the meromorphic function $\frac{1}{f}$. So we obtain $D R\left(\frac{\mathcal{D}}{A n n_{\mathcal{D}}(1 / f)}\right) \simeq D R(\mathcal{O}(\star D))=\Omega^{\bullet}(\star D)$ as it is clear that $\mathcal{D} \cdot \frac{1}{f} \simeq \frac{\mathcal{D}}{A n n_{\mathcal{D}}(1 / f)}$.

The crucial step is then the comparison between the left ideals $A n n_{\mathcal{D}}^{(1)}(1 / f)$ and $A n n_{\mathcal{D}}(1 / f)$ : if $A n n_{\mathcal{D}}^{(1)}(1 / f)=A n n_{\mathcal{D}}(1 / f)$ we obtain that

$$
\begin{aligned}
D R\left(\widetilde{M}^{\log D}\right) & =D R\left(\frac{\mathcal{D}}{A n n_{\mathcal{D}}^{(1)}(1 / f)}\right) \\
& =D R\left(\frac{\mathcal{D}}{A n n_{\mathcal{D}}(1 / f)}\right) \simeq \Omega^{\bullet}(\star D) .
\end{aligned}
$$

For the computational aspects, we note:

1. The free divisor $D$ must be Spencer. This condition can be tested using Gröbner bases in the Weyl algebra to compute the modules of the syzygies that appear, computing a free resolution of $M^{\log D}$ and checking whether each module is the one required by the Spencer resolution presented in Definition 2.1. We used the package for $\mathcal{D}$-modules, Macaulay 2 ([Grayson et al. 99]), written by A. Leykin and $\mathrm{H}$. Tsai.

2. The comparison between $A n n_{\mathcal{D}}(1 / f)$ and $A n n_{\mathcal{D}}^{(1)}(1 / f)$ needs the computation of the annihilator. We have used [Noro 02] in the examples.

3. By [Torrelli 04, Proposition 1.3], if $A n n_{\mathcal{D}}^{(1)}(1 / f)=$ $A n n_{\mathcal{D}}(1 / f)$ then the smallest integer root of the $b$ function $b_{f}$ is -1 . The global $b$-function can be computed with the algorithms of [Oaku 97] or [Noro 02]. We have used for the examples the powerful implementation of the latter in [Noro et al. 00].

With respect to the roots of the $b$-function of free divisors, we can query the following:

Problem 3.2. Let $D \equiv(f=0)$ be a free divisor. Is -1 the smallest integer root of $b_{f}$ ?

In Figure 1 we give examples of free divisors with their global $b$-functions and their Euler vector fields (i.e., of type $\chi=\sum w_{i} x_{i} \partial_{x_{i}}$ with $w_{i} \geq 0$ and $\chi(f)=c \cdot f$ for some $c \in \mathbf{C} \backslash\{0\})$. Criterion 3.1 applies for all of them, so LCT holds.

The examples in our list have been selected because they belong to a family that we are about to define.

Definition 3.3. We will say that a divisor $D \subset X$ is weakly locally quasi-homogeneous if for all $x \in D$ there are local coordinates $\left(z_{1}, \ldots, z_{n}\right)$ on $X$, centered at $x$, with respect to which $D$ has a defining equation $h\left(z_{1}, \ldots, z_{n}\right) \in \mathcal{O}$ such that $h\left(z_{1}^{w_{1}}, \ldots, z_{n}^{w_{n}}\right)$ is homogeneous of strictly positive weight with the weights $w_{i}$ positive or (not all) zero.

This is the case for our examples in Figure 1. They have weight 0 for the variable $z$ and the singular locus is the $z$-axis. It is clear that the change $z \mapsto z+\alpha$ $(\alpha \in \mathbf{C})$ produces a equation that admits the same set of weights. We have found many more examples of weakly locally quasi-homogeneous divisors, and it is apparent that they always verify LCT. We dare to propose the next conjecture based on this experimental evidence. 


\begin{tabular}{|c|c|c|}
\hline (Local) Equation $f$ & Euler operator & Global $b$-function $b_{f}$ \\
\hline$x y(x+y)(x z+y)$ & $x \partial_{x}+y \partial_{y}$ & $(s+3 / 4)(s+1 / 2)(s+5 / 4)(s+1)^{3}$ \\
\hline$x y(x+y)(x-y)(x z+y)$ & $x \partial_{x}+y \partial_{y}$ & $(s+1)^{3}(s+6 / 5)(s+3 / 5)(s+2 / 5)(s+4 / 5)$ \\
\hline$y\left(x^{2}+y\right)\left(x^{2} z+y\right)$ & $x \partial_{x}+2 y \partial_{y}$ & $\begin{array}{c}(s+4 / 3)(s+5 / 6)(s+1)^{3} \\
(s+1 / 2)(s+2 / 3)(s+7 / 6)\end{array}$ \\
\hline$(x z+y)\left(x^{3}-y^{3}\right)$ & $x \partial_{x}+y \partial_{y}$ & $(s+3 / 4)(s+1 / 2)(s+5 / 4)(s+1)^{3}$ \\
\hline$(x z+y)\left(x^{4}-y^{4}\right)$ & $x \partial_{x}+y \partial_{y}$ & $(s+1)^{3}(s+6 / 5)(s+3 / 5)(s+2 / 5)(s+4 / 5)$ \\
\hline$(x z+y)\left(x^{7}-y^{7}\right)$ & $x \partial_{x}+y \partial_{y}$ & $\begin{array}{c}(s+1 / 2)(s+7 / 8)(s+9 / 8)(s+5 / 8) \\
(s+3 / 4)(s+1)^{3}(s+3 / 8)(s+1 / 4)\end{array}$ \\
\hline$x y\left(x^{2}+y^{3}\right)\left(x^{2} z+y^{3}\right)$ & $3 x \partial_{x}+2 y \partial_{y}$ & $\begin{array}{c}(s+20 / 17)(s+7 / 17)(s+23 / 17)(s+16 / 17) \\
(s+13 / 17)(s+5 / 17)(s+14 / 17)(s+12 / 17) \\
(s+10 / 17)(s+18 / 17)(s+11 / 17)(s+1)^{3} \\
(s+15 / 17)(s+8 / 17)(s+21 / 17)(s+9 / 17)(s+19 / 17)\end{array}$ \\
\hline
\end{tabular}

FIGURE 1. Free divisors that verify LCT.

Conjecture 3.4. If $D$ is a weakly locally quasihomogeneous free divisor, then LCT holds.

Proving LCT holds for locally quasi-homogeneous free divisors, makes strong use of the fact that such divisors have a structure of an analytical product around any point of the divisor. Our examples do not have this property, so the proof of Conjecture 3.4 has to be more subtle. We think that one of the ways in which the hypotheses of the locally quasi-homogeneous case could be relaxed is that some of the weights can be zero. The condition on the existence of a local weighted equation seems to be more complex to relax; in the next section we will give examples of free divisors that are defined by weighted homogeneous equations (with all the weights positive) but do not verify LCT. Thus, by [Castro et al. 96], they are not locally quasi-homogeneous (see Remark 5.8).

\section{HOW TO DEDUCE THAT LCT DOES NOT HOLD}

We shall state here a criterion giving a sufficient condition to deduce that LCT does not hold for a given free divisor. This criterion is the converse of Criterion 3.1.
Criterion 4.1. If $D$ is a Spencer divisor, then $A n n_{\mathcal{D}}(1 / f) \neq A n n_{\mathcal{D}}^{(1)}(1 / f) \Rightarrow$ LCT does not hold for $D$.

Proof: Let us consider the natural sequence of $\mathcal{D}$-module morphisms

$$
0 \longrightarrow K \longrightarrow \widetilde{M}^{\log D} \stackrel{\phi_{D}}{\longrightarrow} \mathcal{O}(\star D)
$$

where $\phi_{D}(\bar{P})=P\left(\frac{1}{f}\right)$ for each $\bar{P} \in \widetilde{M}^{\log D}$ and $K$ is the kernel of $\phi_{D}$. We have

$$
K=\frac{A n n_{\mathcal{D}}(1 / f)}{A n n_{\mathcal{D}}^{(1)}(1 / f)} .
$$

Suppose LCT holds for $D$. Then the inclusion map $i_{D}: \Omega^{\bullet}(\log D) \rightarrow \Omega^{\bullet}(\star D)=D R(\mathcal{O}(\star D))$ is a quasiisomorphism. On the other hand, since $D$ is Spencer, we have $D R\left(\widetilde{M}^{\log D}\right) \simeq \Omega^{\bullet}(\log D)$ (see [Castro and Ucha 02, Theorem 4.3]). Then the composition morphism

$$
\psi: D R\left(\widetilde{M}^{\log D}\right) \simeq \Omega^{\bullet}(\log D) \stackrel{i_{D}}{\rightarrow} \Omega^{\bullet}(\star D)=D R(\mathcal{O}(\star D))
$$


is a quasi-isomorphism. We have that $\psi=D R\left(\phi_{D}\right)$ by [Calderón and Narváez 04]; thus, by Proposition 4.2, $\phi_{D}$ must be an isomorphism, contradicting that $K \neq(0)$.

Proposition 4.2. [Mebkhout 89, Chapter II, Theorem 4.1.5] Suppose $\phi: M \rightarrow M^{\prime}$ is a morphism of holonomic $\mathcal{D}$-modules. If $D R(\phi): D R(M) \rightarrow D R\left(M^{\prime}\right)$ is a quasiisomorphism, then $\phi$ is an isomorphism.

Proof: Taking the kernel and cokernel of $\phi$, it is enough to show that if $M$ is a holonomic $\mathcal{D}$-module such that $D R(M)=0$, then $M=0$. So, suppose we have $D R(M)=0$ for a holonomic $\mathcal{D}$-module $M$. By [Mebkhout 89, page 41] we have $\operatorname{Sol}\left(M^{*}\right) \simeq D R(M)$ and then $\operatorname{Sol}\left(M^{*}\right)=0$. By applying [Mebkhout 89, Chapter II, Theorem 4.1.5], we get $\mathcal{D}^{\infty} \otimes_{\mathcal{D}} M^{*}=0$ where $\mathcal{D}^{\infty}$ is the ring of linear differential operators of infinite order. Since $\mathcal{D}^{\infty}$ is faithfully flat over $\mathcal{D}$ (see [Sato et al. 73, Theorem 3.4.1.]), we get $M^{*}=0$ and then $M=0$.

Criteria 3.1 and 4.1 give a necessary and sufficient condition to decide if LCT holds for a Spencer free divisor. In particular, we have proved Conjecture 1.1 for Spencer divisors:

Theorem 4.3. Let $D$ be a Spencer free divisor. LCT holds for the germ $(D, x)$ if and only if the annihilator ideal $A n n_{\mathcal{D}_{x}}(1 / f)$ is generated by elements of order 1 , where $f$ is a local equation of the germ $(D, x)$.

\section{ON THE REGULARITY OF LOGARITHMIC D-MODULES}

To apply Criterion 4.1 we have chosen an alternative way that, at the same time, treats the following interesting problem:

Problem 5.1. Are the logarithmic $\mathcal{D}$-modules $M^{\log D}$ and $\widetilde{M}^{\log D}$ regular holonomic for any free divisor $D$ ?

The answer is yes for plane curves (see [Ucha 99] and [Castro and Ucha 01]) and for all the examples we have studied, including non-Euler homogeneous examples. Moreover, for any Spencer divisor $D$, since $\left(M^{\log D}\right)^{*}$ is isomorphic to $\widetilde{M}^{\log D}$ (see Theorem 2.4), the regularity of $M^{\log D}$ is equivalent to the one of $\widetilde{M}^{\log D}$.

The module $\mathcal{O}(\star D)$ is regular holonomic (see, for example, [Mebkhout 89, Chap. II, Th. 2.2.4]) so it is enough, in order to prove the regularity of $\widetilde{M}^{\log D}$, to prove the same property for the kernel $K$ of the natural map

$$
\widetilde{M}^{\log D} \stackrel{\phi_{D}}{\longrightarrow} \mathcal{O}(\star D) .
$$

Proving the regularity of a $\mathcal{D}$-module, computationally, is a very difficult problem in general. We have found many tractable examples due to a friendly presentation of $K$ that we have obtained for our examples in Figure 2. These proposed free divisors are particular cases of the family $\left\{D_{p, q} \equiv\left((x z+y)\left(x^{p}-y^{q}\right)=0\right) \subset \mathbf{C}^{3}\right\}$ for $p, q \in \mathbf{N}$. They are Koszul free ${ }^{3}$, and therefore Spencer. Criterion 4.1 applies, so they do not verify LCT. It is interesting to point out that every element of the family admits an Euler vector field $E=q x \partial_{x}+p y \partial_{y}+(p-q) z \partial_{z} \in$ $\operatorname{Der}\left(-\log D_{p, q}\right)$ with strictly positive weights for all the variables when $p>q$. This fact means that the defining equation is a weighted homogeneous polynomial.

Remark 5.2. It would be interesting to describe the $b$ functions for all the elements of the family. The roots seems to follow a pattern related to the weights of the variables, in a way somewhat analogous to the isolated (quasi-homogeneous) singularity case.

Let us explain how we have studied the regularity of the kernel $K$ for the divisors of the family $\left\{D_{p, q} \equiv\right.$ $\left.\left.(x z+y)\left(x^{p}-y^{q}\right)=0\right)\right\}$. To obtain a presentation of the quotient

$$
K=\frac{A n n_{\mathcal{D}}(1 / f)}{A n n_{\mathcal{D}}^{(1)}(1 / f)}
$$

the following procedure is well known:

1. Get a set of generators $\left\{g_{1}, \ldots, g_{r}\right\}$ of $A n_{\mathcal{D}}(1 / f)$ and a set of generators $\left\{l_{1}, \ldots, l_{s}\right\}$ of $A n n_{\mathcal{D}}^{(1)}(1 / f)$.

2. Compute the $\mathcal{D}$-module $\mathcal{S}$ of syzygies among $g_{1}, \ldots, g_{r}, l_{1}, \ldots, l_{s}$.

3. For every generator $\mathrm{s} \in \mathcal{D}^{r+s}$ of $\mathcal{S}$ delete its last $s$ components to obtain $\widehat{\mathbf{s}} \in \mathcal{D}^{r}$.

In this way, if $S=\left\langle\mathrm{s}_{1}, \ldots, \mathrm{s}_{t}\right\rangle$ then we have

$$
K \simeq \frac{\mathcal{D}^{r}}{\left\langle\widehat{\mathbf{s}}_{1}, \ldots, \widehat{\mathbf{s}}_{t}\right\rangle} ;
$$

that is, using a matrix of $r$ rows and $t$ columns (each column is a generator $\widehat{\mathbf{s}}_{i}$ ).

As soon as $p$ and $q$ grow for $D_{p, q}$ (for $p, q \geq 8$ ), the computations of annihilators, $b$-functions, and kernels become huge and the examples intractable with the implementation we have used. However, in the tractable cases

\footnotetext{
${ }^{3} \mathrm{An}$ argument about the dimension of the characteristic variety of $\widetilde{M}^{\log D_{p, q}}$ can be used for the whole family.
} 


\begin{tabular}{|c|c|}
\hline (Local) Equation $f$ & Global $b$-function $b_{f}$ \\
\hline & \\
$(x z+y)\left(x^{4}-y^{3}\right)$ & $(s+19 / 15)(s+2 / 3)(s+1 / 2)(s+1)^{3}(s+13 / 15)$ \\
& $(s+17 / 15)(s+4 / 3)(s+16 / 15)(s+14 / 15)(s+5 / 4)$ \\
$(s+7 / 15)(s+3 / 4)(s+8 / 15)(s+11 / 15)$
\end{tabular}

FIGURE 2. Free divisors that do not verify LCT.

the kernels turn out to be represented by matrices with a special structure. We have used [Grayson et al. 99] and [Noro et al. 00] $]^{4}$.

Example 5.3. In the case $p=4$ and $q=3$, the kernel $K$ can be represented (using some elementary simplifications) by the matrix

$$
\left(\begin{array}{cccccc}
x & y & z \partial_{z}+8 & \frac{3}{4} \partial_{x} & -\frac{9}{16} \partial_{x}^{2} & P \\
0 & 0 & 0 & 1 & 0 & 0 \\
0 & 0 & 0 & 0 & 1 & 0 \\
0 & 0 & 0 & 0 & 0 & 1
\end{array}\right)
$$

where $P=\frac{27}{64} z \partial_{x}^{3}+\frac{64}{3} z \partial_{y}^{2} \partial_{z}-100 \partial_{x} \partial_{y} \partial_{z}-\frac{28}{3} \partial_{y}^{2}$.

Example 5.4. In the case $p=5$ and $q=3$, the kernel can be represented by

$$
\left(\begin{array}{ccccc}
x & y & z \partial_{z}+5 & P & Q \\
0 & 0 & 0 & 1 & 0 \\
0 & 0 & 0 & 0 & 1
\end{array}\right)
$$

\footnotetext{
${ }^{4}$ More precisely, we have computed $A n n_{\mathcal{D}}(1 / f)$ computing the annihilator of $f^{s}$ in $\mathcal{D}[s]$ with the command AnnFs of [Grayson et al. 99] and replaced $s$ by -1 , provided that Risa/Asir (with the command bfct) has shown that the smallest integer root of $b_{f}$ is -1 .
}

where

$$
\begin{aligned}
P= & -36 \partial_{z}^{2}, \\
Q= & -\frac{48}{5} z^{2} \partial_{y} \partial_{z}^{2}+\frac{3204}{125} z \partial_{x} \partial_{z}^{2}-\frac{216}{25} z \partial_{y} \partial_{z} \\
& +\frac{17397}{125} \partial_{x} \partial_{z}-\frac{417}{125} \partial_{y} .
\end{aligned}
$$

Lemma 5.5. If the $\mathcal{D}$-module $K$ is represented by the matrix

$$
\mathcal{M}=\left(\begin{array}{ccccccc}
x & y & z \partial_{z}+a & P_{1} & P_{2} & \cdots & P_{t} \\
0 & 0 & 0 & 1 & 0 & \cdots & 0 \\
0 & 0 & 0 & 0 & 1 & \cdots & 0 \\
\vdots & \vdots & \vdots & \vdots & \vdots & & \vdots \\
0 & 0 & 0 & 0 & 0 & \cdots & 1
\end{array}\right),
$$

for any $a \in \mathbf{C}$ and $P_{1}, \ldots, P_{t} \in \mathcal{D}$, then

$$
K \simeq \mathcal{D} /\left\langle x, y, z \partial_{z}+a\right\rangle
$$

Proof: It is enough to define the isomorphism $\varphi$

$$
\mathcal{D}^{t+1} / N \stackrel{\varphi}{\longrightarrow} \mathcal{D} /\left\langle x, y, z \partial_{z}+a\right\rangle,
$$


where $N$ is the submodule generated by the columns of $\mathcal{M}$. It is defined as follows:

$$
\begin{array}{cc}
\overline{\mathbf{e}_{1}} & \mapsto \overline{1} \\
\overline{\mathbf{e}_{2}} & \mapsto \overline{-P_{1}} \\
\vdots & \vdots \\
\overline{\mathbf{e}_{t+1}} & \mapsto \overline{-P_{t}}
\end{array}
$$

and its inverse maps $\overline{1}$ to $\overline{\mathbf{e}_{1}}$.

Lemma 5.6. If the $\mathcal{D}$-module $K$ admits a presentation as in Lemma 5.5, then it is regular holonomic.

Proof: Obvious from the representation we have obtained for $K \simeq \mathcal{D} /\left\langle x, y, z \partial_{z}+a\right\rangle$, clearly a regular holonomic $\mathcal{D}$ module.

Theorem 5.7. The elements of the family $\left\{D_{p, q}\right\}$ with kernels as in Lemma 5.5 do not verify LCT. In addition, the corresponding $\mathcal{D}$-modules $M^{\log D_{p, q}}$ and $\widetilde{M}^{\log D_{p, q}}$ are regular holonomic.

Proof: Simply apply Criterion 4.1; the corresponding kernels are not null so LCT does not hold. The regularity is a consequence of Lemma 5.6.

Remark 5.8. In [Calderón and Narváez 02, Problem 6.5] the authors ask whether a free divisor defined by a quasihomogeneous polynomial (with strictly positive weights) is locally quasi-homogeneous. The answer to this question is negative: by Theorem 5.7 the first three examples provided in Figure 2 are free divisors defined by quasihomogeneous polynomials (with strictly positive weights) that do not verify LCT. Since locally quasi-homogeneous free divisors verify LCT (see [Castro et al. 96]), these divisors are not locally quasi-homogeneous. We think that every element of the family with $p>q(\operatorname{lcm}(p, q)=1)$ are in the same situation, but we don't have a proof for this general result.

In [Castro et al. 96] it was noted that if LCT holds then the morphism

$$
d_{1}: \check{H}^{n-1}(V \backslash 0, \mathcal{O}) \rightarrow \breve{H}^{n-1}\left(V \backslash 0, \Omega^{1}(\log D)\right)
$$

is injective, where $V$ is a Stein neighborhood (sufficiently small) of 0 . In dimension 2, this condition is equivalent to LCT (see [Calderón et al. 02]).

The examples of Figure 2 show that the condition on $d_{1}$ is not sufficient in dimension greater than 2. If $\left\{\omega_{1}, \ldots, \omega_{n}\right\}$ is a free basis of $\Omega^{1}(\log D)$ as $\mathcal{O}_{V}$-module and $\delta_{1}, \ldots, \delta_{n}$ is the dual basis of $\operatorname{Der}(-\log D)$, then

$$
\check{H}^{n-1}\left(V \backslash 0, \mathcal{O}_{\mathbf{C}^{n}}\right) \simeq \check{H}^{n-1}\left(V \backslash 0, \Omega^{0}(\log D)\right)
$$

and

$$
\check{H}^{n-1}\left(V \backslash 0, \mathcal{O}_{\mathbf{C}^{n}}\right)^{n} \simeq \check{H}^{n-1}\left(V \backslash 0, \Omega^{1}(\log D)\right) .
$$

The morphism $d_{1}$ can be read now as

$$
\begin{array}{ccc}
\check{H}^{n-1}\left(V \backslash 0, \mathcal{O}_{\mathbf{C}^{n}}\right) & \stackrel{d_{1}}{\rightarrow} & \check{H}^{n-1}\left(V \backslash 0, \mathcal{O}_{\mathbf{C}^{n}}\right)^{n} \\
{[g]} & \mapsto & \left(\left[\delta_{1} \cdot g\right], \ldots,\left[\delta_{n} \cdot g\right]\right)
\end{array} .
$$

The space $\breve{H}^{n-1}\left(V \backslash 0, \mathcal{O}_{\mathbf{C}^{n}}\right)$ is isomorphic to the space $\mathcal{S}$ of Laurent series, convergent for all $\underline{x}=\left(x_{1}, \ldots, x_{n}\right)$ with $\underline{x} \neq 0$ and whose nonzero coefficients are those with strictly negative indices in all variables. It is clear that if there exists an element $\delta_{1}=\sum_{i=1}^{n} w_{i} x_{i} \partial_{i}$ with all the $w_{i} \geq 0$ in the basis of $\operatorname{Der}(-\log D)$ it follows that $d_{1}$ is injective, as in the first three examples of Figure 2.

\section{CONCLUSIONS}

Many examples have been treated with the explicit methods we have proposed in this work to study the Logarithmic Comparison Theorem (LCT) on free divisors. The results on the weakly locally quasi-homogeneous examples we have tried has lead us to conjecture that they verify LCT. As a consequence, they would be Spencer divisors.

We have proved that LCT holds for a Spencer free divisor $D \equiv(f=0)$ if and only if $A n n_{\mathcal{D}}(1 / f)$ is generated by differential operators of order 1 .

On the other hand, we have given examples that answer a question proposed in [Calderón and Narváez 02]: whether there exist free divisors defined by weighted homogeneous polynomials that are not locally quasihomogeneous. We have proved that for these examples the logarithmic $\mathcal{D}$-modules are regular.

\section{ACKNOWLEDGMENTS}

Both authors were partially supported by BFM-2001-3164 and FQM-333. We are very grateful to Professors L. NarváezMacarro and Z. Mebkhout for their useful comments. During the preparation of the final version of this work, the first author was visting the École Normale Supérieure (Paris). He is grateful to the Département de Mathématiques et Applications for its hospitality. 


\section{REFERENCES}

[Bernstein 72] J. Bernstein. "Analytic Continuation of Generalized Functions with Respect to a Parameter." Funkcional. Anal. i Priložen 6:4 (1972), 26-40.

[Calderón 99] F. J. Calderón-Moreno. "Logarithmic Differential Operators and Logarithmic De Rham Complexes Relative to a Free Divisor." Ann. Sci. E.N.S. 32:4 (1999), 701-714.

[Calderón et al. 02] F. J. Calderón-Moreno, D. Mond, L. Narváez-Macarro, and F. J. Castro-Jiménez. "Logarithmic Cohomology of the Complement of a Plane Curve." Comment. Math. Helv. 77:1 (2002), 24-38.

[Calderón and Narváez 02] F. J. Calderón-Moreno and L. Narváez-Macarro. "The Module $D \cdot f^{s}$ for Locally QuasiHomogeneous Free Divisors." Compositio Math. 134 (2002), 59-74.

[Calderón and Narváez 04] F. J. Calderón-Moreno and L. Narváez-Macarro, "Dualité et comparaison sur les complexes de de Rham logarithmiques par rapport aux diviseurs libres." Preprint, 2004.

[Castro et al. 96] F. J. Castro-Jiménez, D. Mond, and L. Narváez-Macarro. "Cohomology of the Complement of a Free Divisor." Trans. Amer. Math. Soc. 348:8 (1996), 3037-3049.

[Castro and Ucha 01] F. J. Castro-Jiménez, and J. M. UchaEnríquez. "Explicit Comparison Theorems for $\mathcal{D}$ Modules." J. Symbolic Comput. 32:6 (2001), 677-685.

[Castro and Ucha 02] F. J. Castro Jiménez and J. M. Ucha Enríquez. "Free Divisors and Duality for DModules." Proc. Steklov Inst. of Math. 238 (2002), 88-96.

[Grayson et al. 99] D. Grayson and M. Stillman "Macaulay2: A Software System for Research in Algebraic Geometry." Available from World Wide Web (http://www.math.uiuc.edu/Macaulay2), 1999.

[Grothendieck 66] A. Grothendieck. "On the de Rham Cohomology of Algebraic Varieties." Publ. Math. de l'I.H.E.S. 29 (1966), 95-103.
[Leykin and Tsai 01] A. Leykin and H. Tsai. "D-Module Package for Macaulay 2." Available from World Wide Web (http://www.math.cornell.edu/ ${ }^{\sim}$ htsai), 2001.

[Mebkhout 89] Z. Mebkhout. Le formalisme des six opérations de Grothendieck pour les $\mathcal{D}_{X}$-modules cohérents, Travaux en Cours, 35. Paris: Hermann, 1989.

[Noro 02] M. Noro. "An Efficient Modular Algorithm for Computing the Global b-Function." In Mathematical Software (Beijing, 2002), pp. 147-157. River Edge, NJ: World Sci. Publishing, 2002.

[Noro et al. 00] M. Noro, T. Shimoyama, and T. Takeshima. "A Computer Algebra System Risa/Asir." Available from World Wide Web (ftp://archives.cs.ehime -u.ac.jp/pub/asir2000/), 2000.

[Oaku 97] T. Oaku. "Algorithms for the b-Function and $\mathcal{D}$ Modules Associated with a Polynomial." Journal of Pure and Applied Algebra 117/118 (1997), 495-518.

[Sato et al. 73] M. Sato, T. Kawai, and M. Kashiwara. "Microfunctions and Pseudo-Differential Equations." In $\mathrm{Hy}$ pergeometric and Pseudo-Differential Equations, Lecture Notes in Math., 287, pp. 265-529. Berlin: Springer, 1973.

[Saito 80] K. Saito. "Theory of Logarithmic Differential Forms and Logarithmic Vector Fields." J. Fac Sci. Univ. Tokyo 27 (1980), 256-291.

[Saito et al. 00] M. Saito, B. Sturmfels, and N. Takayama. Gröbner Deformations of Hypergeometric Differential Equations, Algorithms and Computation in Mathematics, 6. Berlin: Springer-Verlag, 2000.

[Torrelli 98] T. Torrelli.. "Equations fonctionnelles pour une fonction sur un espace singulier." PhD. diss., Université de Nice-Sopia Antipolis, 1998.

[Torrelli 04] T. Torrelli. "On Meromorphic Functions Defined by a Differential System of Order 1." Bull. Soc. Math. France. To appear, 2004.

[Ucha 99] J. M. Ucha-Enríquez. "Métodos constructivos en álgebras de operadores diferenciales." PhD. diss., Universidad de Sevilla, 1999.

F.J. Castro-Jimnez, Departamento de Algebra, Universidad de Sevilla, Avda Reina Mercedes, s.n., 41012 Sevilla, Spain (castro@us.es)

J. M. Ucha-Enrquez, Departamento de Algebra, Universidad de Sevilla, Avda Reina Mercedes, s.n., 41012 Sevilla, Spain (ucha@us.es)

Received October 28, 2003, accepted in revised form May 20, 2004. 